\title{
Reconocimiento de diásporas de Malveae (Malvaceae) en muestras de suelos de zonas serranas (Sierras Chicas, Córdoba, Argentina) afectadas por incendios
}

\author{
Malveae (Malvaceae) diaspore recognition in soil samples from montane areas \\ (Sierras Chicas, Córdoba, Argentina) affected by fire
}

Marta Ester Carreras ${ }^{1,2}$, Elsa Fuentes ${ }^{1}$, Jimena Elisa Martinat ${ }^{1}$ \& Lucas Manuel Carbone $^{1}$

\begin{abstract}
Resumen
En el marco de un estudio de regeneración post-incendio de la vegetación autóctona en campos de sierra ubicados en proximidades de Falda del Carmen (Sierras Chicas, Córdoba, Argentina), se ha analizado el banco de semillas aéreo para facilitar la identificación de las especies presentes en las muestras de suelo. Entre las familias con mayor diversidad y abundancia en la zona evaluada, las Malvaceae se encuentran representadas por 14 especies pertenecientes a los géneros: Abutilon Mill., Gaya Kunth., Krapovickasia Fryxell, Malvastrum A. Gray, Pavonia Cav., Pseudabutilon R. E. Fr., Sida L. y Sphaeralcea A. St.-Hil.. Se presentan dos claves dicotómicas para diferenciar las especies utilizando caracteres morfológicos de las diásporas, mericarpos y semillas respectivamente, acompañadas por las descripciones y las ilustraciones de las estructuras consideradas. Se tienen en cuenta aspectos morfológicos de los mericarpos (forma, tamaño, superficie de las caras dorsal y laterales, dehiscencia, aristas, pubescencia, divisiones internas, número de semillas por mericarpo) y de las semillas (forma, tamaño, superficie, pubescencia, hilo).

Palabras-clave: Malvaceae, diásporas, clave dicotómica, banco de semillas, áreas post-fuego.
\end{abstract}

\begin{abstract}
As part of a study of post-fire regeneration of native vegetation in montane areas near Falda del Carmen (Sierras Chicas, Córdoba, Argentina), we analyzed the aerial seed bank to help identify the species present in soil samples. Among families with greater diversity and abundance in the study area, the Malvaceae are represented by 14 species belonging to the genera: Abutilon, Gaya, Krapovickasia, Malvastrum, Pavonia, Pseudabutilon, Sida and Sphaeralcea. Two dichotomous keys are presented to differentiate the species using the morphological characters of diaspores, mericarps and seeds, respectively, accompanied by descriptions and illustrations of the structures examined. These keys take into account mericarp morphology (shape, size, dorsal and lateral surface, dehiscence, cusps, pubescence, internal divisions, number of seeds per mericarp) and seed morphology (size, shape, surface, pubescence, hilum).
\end{abstract}

Key words: Malvaceae, diaspore, dichotomous key, seed bank, post-fire area.

\section{Introducción}

Las Sierras de Córdoba, ubicadas en el centro del sector continental americano de Argentina, están formadas por cordones montañosos de mediana altura, entre 500 y casi $3.000 \mathrm{~m}$, que corren de Norte a Sur. Las denominadas Sierras Chicas, constituyen el cordón más oriental, con un máximo de $1950 \mathrm{~m}$ de altitud. Las variaciones en altitud, desde las lomadas menores hasta las cumbres mayores, determina la presencia de pisos de vegetación, entre los que se individualizan en forma ascendente: el bosque serrano, el romerillal o arbustal y los pastizales; cuya existencia, amplitud y elevación está condicionada por otros factores como la latitud, orientación geográfica, biología de la dispersión, naturaleza del suelo, relieve y clima en general, y por la intervención de otros como la tala de los bosques y el fuego (Luti et al. 1979).

\footnotetext{
${ }^{1}$ Universidad Nacional de Córdoba, Facultad de Ciencias Agropecuarias, Herbario ACOR, CC 509. 5000, Córdoba, Argentina.

${ }^{2}$ Autora para correspondência: mcarrera@agro.unc.edu.ar
} 
En general, estos ambientes se encuentran muy degradados y expuestos a un gran número de presiones que van reduciendo cada vez más su biodiversidad (Montenegro 2010). Las actividades extractivas de madera, leña, minerales, hierbas medicinales, aromáticas y fauna, conjuntamente con el sobrepastoreo, los incendios de campos, provocados o accidentales y el turismo, han conducido a un deterioro marcado de la vegetación original, contribuyendo a modelar el paisaje actual (Verzino et al. 2005).

En la historia de los biomas del mundo, los incendios aparecen como un componente abiótico que actúa balanceando el equilibrio entre las especies. La mayor frecuencia de incendios tiene un origen antrópico y, en pequeña escala, son resultado de eventos naturales (Abril \& González 1999). En Córdoba, aproximadamente el 30\% del área serrana se quema anualmente; los incendios se centran en el sector occidental con predominio de vegetación xerófíla (Martínez Carretero 1995). Durante los años 2008 y 2009 se han registrado incendios que han afectado hasta 100.000 ha de las sierras cordobesas (Estadística de Incendios Forestales 2008, 2009).

La capacidad de germinación y de rebrote de las especies permite predecir la recuperación de la vegetación después de un incendio (Díaz-Delgado 2003). Para evaluar y conservar la biodiversidad, considerada como un patrimonio de riqueza genética, se debe tener en cuenta, entre otros, la información oculta en el banco de semillas, la cual forma parte de la flora del lugar y constituye una fase crucial en el recambio de las poblaciones y la dinámica de las comunidades vegetales (Ferri et al. 2009).

Para analizar el banco de semillas del suelo se utilizan métodos de separación, de germinación o rara vez, de enterramiento. De todos ellos, el método directo es más efectivo que el indirecto (Piudo \& Cavero 2005). Las semillas se aíslan por tamizado o flotación y luego se identifican por comparación con una colección de semillas realizada previamente con material de la zona, o mediante el uso de claves especializadas (Ferri et al. 2009, Piudo \& Cavero 2005).

Si bien contribuciones como las de Martin \& Barkley (1973), Petetin \& Molinari (1982), Bianco et al. (2000) y Meyer \& Effenberger (2007) resultan útiles para la identificación de algunas especies, surge la necesidad de contar con claves de los grupos mejor representados, dado que las diferencias específicas suelen ser sutiles y difíciles de observar.

En la zona de estudio, las Malvaceae constituyen la cuarta familia en abundancia y diversidad, precedidas por las Poaceae, Asteraceae y Fabaceae (Fuentes et al. 2009a, b; Martinat et al. 2009).

Malvaceae es una familia de distribución cosmopolita con 243 géneros y alrededor de 4.300 especies (Bayer \& Kubitzki, 2003), representada en Argentina por 198 especies agrupadas en 35 géneros (Krapovickas, 1999).

Bayer et al. (1999) dividieron a la familia en 9 subfamilias, de las cuales Malvoideae es considerada por Hinsley (2006) un grupo cladísticamente troncal (Malvaceae s.s.) compuesto por varias tribus: Gossypieae, Hibisceae, Kydieae y Malveae (Bayer \& Kubitzki, 2003).

Especies de esta familia están citadas como forrajeras (Fuentes et al. 2010), ornamentales (Carreras et al. 2008, Torres et al. 2008, Barrionuevo 2008), medicinales (Martínez 2005, Arias Toledo 2009), productoras de néctar (Castillo Carrillo 2006) y malezas (Medrano et al. 1999, López Martínez 2009).

En su mayoría poseen frutos esquizocárpicos, generalmente rodeados por el cáliz persistente, que se desarticulan a la madurez en mericarpos indehiscentes o dehiscentes, por lo que la forma de dispersión de las especies es mediante mericarpos o semillas libres (Carreras et al. 2009).

Con el objeto de facilitar la identificación, en muestras de suelo, de diásporas (mericarpos y semillas) de especies de Malvaceae que crecen en zonas serranas de Córdoba afectadas por incendios, se presentan dos claves dicotómicas basadas en la morfología de mericarpos y de semillas respectivamente, acompañadas por descripciones e ilustraciones.

\section{Materiales y Métodos}

\section{Área de estudio}

La zona de estudio comprende alrededor de 3.000 ha ubicadas en las laderas orientales de las Sierras Chicas, en las proximidades de Falda del Carmen, Dep. Santa María, Córdoba, Argentina $\left(31^{\circ} 28^{\prime}-31^{\prime} \mathrm{S}, 64^{\circ} 26^{\prime}-31^{\prime} \mathrm{O}\right)$. Esta área ha sido perturbada por sucesivos incendios; el último se registró en octubre de 2005 y afectó los estratos herbáceo, arbustivo y arbóreo, siendo los dos últimos los que están requiriendo mayor tiempo para su recuperación. 
El relieve es de sierras bajas (500-900 m.s.m.), con pendientes de $16^{\circ}$ a $18^{\circ}$ (Fuentes et al. 2009b). Los suelos son de escaso desarrollo, apoyados sobre un material lítico o paralítico de textura arenosa franca, con el $80 \%$ de la superficie del área ocupada por roca aflorante y bloques dispersos de distintos tamaños. El clima es de dominio semi-húmedo en verano $\left(28-36^{\circ} \mathrm{C}\right)$ y semi-seco en invierno $\left(8-14^{\circ} \mathrm{C}\right)$ con frecuentes heladas $\left(-2\right.$ a $\left.-6^{\circ} \mathrm{C}\right)$. La precipitación anual varía entre 700-900 $\mathrm{mm}$, decrece hacia el oeste y crece en altitud. Los vientos del NE y E predominan de agosto a marzo y los del $\mathrm{O}$ en invierno. La actividad antrópica predominante es la ganadería de cría, seguida por actividades agrícolas y explotación de canteras (Atala et al. 2005).

La vegetación natural corresponde a una zona de contacto entre elementos del bosque chaqueño que asciende por el piedemonte y el bosque serrano que se desarrolla en las laderas (Atala et al. 2005). Los pastos y los arbustos constituyen las dos formas biológicas más representadas en el área. En el estrato arbustivo se encuentra Flourensia oolepis, Acacia caven, Acacia aroma, Aloysia gratissima, Lantana grisebachii, Lippia junelliana, Ruprechtia apetala, Ximenia americana y Schinus fasciculatus, acompañado por especies herbáceas, en su mayoría pertenecientes a las familias Poaceae, Asteraceae, Fabaceae, Malvaceae, Euphorbiaceae y Lamiaceae. El estrato arbóreo está representado por Lithraea molleoides, Zanthoxylum coco y Aspidosperma quebracho-blanco, las dos últimas sólo en estado de rebrote (Fuentes et al. 2009b).

\section{Metodología}

Se realizaron censos florísticos en laderas quemadas y no quemadas, durante los meses de verano, otoño y primavera de 2008, 2009 y 2010, utilizando transectas lineales a lo largo del gradiente altitudinal y otras perpendiculares al sentido de las anteriores (Fuentes et al. 2009b). Las especies fueron identificadas utilizando floras regionales y tratamientos taxonómicos (Fryxell 1999, Krapovickas 1949, 1957, 1996, 2005, 2006, Krapovickas \& Cristóbal 1962). Los ejemplares de referencia fueron depositados en el Herbario ACOR (Facultad de Ciencias Agropecuarias, Universidad Nacional de Córdoba), y catalogados en las siguientes colecciones: Colección de Semillas (CS) y Colección A. M. Planchuelo (AMP). La actualización de los nombres científicos y la determinación del status de las especies se efectuó en base al Catálogo de Plantas Vasculares del Cono Sur (Zuloaga et al. 2008).

Para la observación, análisis y comparación de las diásporas (mericarpos y semillas) de las especies de Malvaceae presentes en el banco de semillas aéreo se utilizó un microscopio estereoscópico Carl Zeiss Stemi DV4. Para la elaboración de las claves dicotómicas se tuvieron en cuenta tanto aspectos morfológicos de los mericarpos (forma, tamaño, superficie de las caras dorsal y laterales, dehiscencia, aristas, pubescencia, divisiones internas o endoglosas y número de semillas por mericarpo) como de las semillas (forma, tamaño, superficie, pubescencia, hilo), dado que en las muestras de suelo pueden aparecer las semillas libres o incluidas en los mericarpos, completos o incompletos. Se realizaron descripciones e ilustraciones de las diásporas teniendo en cuenta los caracteres diferenciales de mayor valor diagnóstico, en base a la observación, análisis y comparación de los materiales estudiados, complementada con los aspectos mencionados en la bibliografía consultada. Con el objeto de facilitar la interpretación de la forma de los mericarpos se mencionan los valores medios del largo, ancho en vista lateral y ancho en vista dorsal; de las semillas se consideran el largo y el ancho. Las ilustraciones son dibujos originales realizados por observación directa de los materiales con microscopio estereoscópico.

\section{Resultados y Discusión}

Se identificaron 14 especies nativas pertenecientes a los géneros Abutilon Mill., Gaya Kunth, Krapovickasia Fryxell, Malvastrum A. Gray, Pavonia Cav., Pseudabutilon R. E. Fr., Sida L. y Sphaeralcea A. St.-Hil., de la tribu Malveae (Malvoideae, Malvaceae). Las especies censadas representan el 35\% del total de Malvaceae citadas para la provincia de Córdoba (Krapovickas 2008). Se detallan a continuación las especies evaluadas: Abutilon pauciflorum A. St.-Hil., Gaya parviflora (Phil.) Krapov., Krapovickasia flavescens (Cav.) Fryxell, Malvastrum coromandelianum (L.) Garcke, M. interruptum K. Schum., Pavonia aurigloba Krapov. \& Cristóbal, Pseudabutilon pedunculatum (R. E. Fr.) Krapov., P. virgatum (Cav.) Fryxell, Sida argentina K. Schum., S. dictyocarpa Griseb. ex K. Schum., S. rhombifolia L., S. spinosa L., S. variegata (Griseb.) Krapov., Sphaeralcea cordobensis Krapov. 
Clave para el reconocimiento de mericarpos de Malveae (Malvaceae) presentes en muestras de suelo de zonas de las Sierras Chicas de Córdoba (Argentina) afectadas por incendios

1. Cara dorsal de los mericarpos con carena media prominente.

2. Mericarpos óseos, indehiscentes, uniseminados, notablemente reticulados con celdas profundas, con carena media prolongada en un apículo. Pelos simples, blancos, rígidos, notables, en cara dorsal y laterales Pavonia aurigloba

2'. Mericarpos papiráceos, dehiscentes por la carena media, uni a pluriseminados, lisos, con o sin aristas. Pelos simples y/o estrellados en la cara dorsal, caras laterales glabras.

3. Mericarpos uniseminados, sin aristas, de 6-8 mm long., con endoglosa muy ornamentada. Cara dorsal con pelos simples dispersos, de menos de $1 \mathrm{~mm}$ long. Caras laterales transparentes con nervaduras formando un retículo Gaya parviflora

3'. Mericarpos pluriseminados, biaristados, de 10-15 mm long., sin endoglosa. Cara dorsal con pelos abundantes, simples de 1,5-2 mm long. en la zona de dehiscencia y estrellados más cortos en el resto de la superficie. Caras laterales opacas lisas Abutilon pauciflorum

1'. Cara dorsal de los mericarpos sin carena media prominente, al menos en la base.

4. Mericarpos sin aristas.

5. Cara dorsal tuberculada, con escasos pelos simples o glabra y márgenes prolongados por una membrana transparente. Mericarpos indehiscentes con una semilla muy adherida a la pared del mericarpo Krapovickasia flavescens

5'. Cara dorsal lisa con pelos cortos estrellados y pelos más largos simples o con dos ramas hacia la cara lateral. Mericarpos con dehiscencia ventral, con una semilla no adherida a la pared del mericarpo Malvastrum interruptum

4'. Mericarpos con aristas.

6. Mericarpos con tres aristas, dehiscentes sólo por el margen ventral Malvastrum coromandelianum

6'. Mericarpos con dos aristas, dehiscentes en el ápice.

7. Cara dorsal de los mericarpos reticulada en la base. Pelos simples asociados con la zona de dehiscencia y en las aristas, pelos estrellados en el dorso y/o aristas, abundantes o escasos.

8. Pelos estrellados abundantes en dorso y aristas. Aristas con pelos simples retrorsos. Parte basal de los mericarpos con un pico en el margen ventral

Sida variegata

8'. Pelos estrellados escasos en dorso y aristas. Aristas con pelos simples antrorsos. Parte basal de los mericarpos sin pico en el margen ventral.

9. Mericarpos rectangulares en vista dorsal. Caras laterales tan anchas como largas, con un tenue reticulado de celdas redondeadas. Aristas dirigidas hacia ventral. Pelos de la zona de dehiscencia de igual largo que los de las aristas

Sida rhombifolia

9'. Mericarpos triangulares u ovalados en vista dorsal. Caras laterales más largas que anchas con un reticulado notable, de celdas alargadas. Aristas erguidas. Pelos de la zona de dehiscencia más largos que los de las aristas.

10. Cara dorsal ovalada

Sida dictyocarpa

10'. Cara dorsal subtriangular con el extremo basal terminado en punta.

11. Mericarpos de alrededor de $2 \mathrm{~mm}$ long. excluidas las aristas. Aristas de alrededor de $1,5 \mathrm{~mm}$ long Sida spinosa

11'. Mericarpos de alrededor de 3,5 mm long., excluidas las aristas. Aristas de alrededor de $0,5 \mathrm{~mm}$ long Sida argentina

7'. Cara dorsal de los mericarpos no reticulada. Pelos estrellados distribuidos uniformemente en la cara dorsal. Pelos simples ausentes. 
12. Mericarpos con caras laterales reticuladas en el tercio inferior, divididos internamente en dos compartimentos superpuestos separados por una endoglosa transversal, completa o incompleta.

13. Endoglosa completa. Cara dorsal con una línea transversal notable, coincidiendo con la endoglosa interna. Aristas de aproximadamente 1/6 del largo total del mericarpo. Caras laterales papiráceas, opacas, con retículo tenue

Pseudabutilon pedunculatum

13'. Endoglosa incompleta pequeña. Cara dorsal sin línea transversal. Aristas en forma de mucrones muy pequeños, apenas sobresaliendo de los pelos. Caras laterales membranáceas, transparentes, con retículo notable Sphaeralcea cordobensis

12'. Mericarpos con caras laterales lisas, sin endoglosa Pseudabutilon virgatum

\section{Clave para el reconocimiento de semillas de Malveae (Malvaceae) presentes en muestras de suelo de zonas de las Sierras Chicas de Córdoba (Argentina) afectadas por incendios}

1. Semillas glabras.

2. Semillas comprimidas, arriñonadas. Hilo lateral.

3. Semillas de alrededor de $1,9 \mathrm{~mm}$ long. $\times 1,8 \mathrm{~mm}$ lat. y $1,1 \mathrm{~mm}$ de espesor

Malvastrum coromandelianum

3'. Semillas de alrededor de $1,5 \mathrm{~mm}$ long. $\times 1,2 \mathrm{~mm}$ lat. y $0,8 \mathrm{~mm}$ de espesor

Malvastrum interruptum

2'. Semillas trígonas o globosas. Hilo apical.

4. Semillas globosas de alrededor de 2,0 $\times 1,8 \mathrm{~mm}$........................ Krapovickasia flavescens

4'. Semillas trígonas de alrededor de $1,8 \times 1,1 \mathrm{~mm}$........................................... Sida spinosa

1'. Semillas con pelos abundantes o escasos en toda la superficie o sólo relacionados con la zona hilar.

5. Semillas con pelos estrellados.

6. Pelos solitarios con 2 ramas

Abutilon pauciflorum

6'. Pelos asociados en grupos otorgando a la superficie un aspecto punteado, con más de 3 ramas.

7. Semillas con las caras laterales de color uniforme Pseudabutilon virgatum

7'. Semillas con las caras laterales con una zona más clara en forma de arco Pseudabutilon pedunculatum

5'. Semillas con pelos simples.

8. Pelos distribuidos en toda la superficie seminal.

9. Pelos abundantes y aún más en la zona hilar y a lo largo de 5 costillas. Semilla piramidal de alrededor de $2 \mathrm{~mm}$ long. $\times 1,5 \mathrm{~mm}$ lat. Hilo apical. Gaya parviflora

9'. Pelos escasos. Semillas lenticulares de alrededor de $1 \mathrm{~mm}$ long. $\times 0,8 \mathrm{~mm}$ lat. Hilo lateral Sphaeralcea cordobensis

8'. Pelos agrupados en áreas delimitadas de la superficie seminal.

10. Hilo lateral. Pelos agrupados en 2 mechones uno por encima y otro por debajo de la zona hilar Pavonia aurigloba

10'. Hilo apical. Pelos exclusivamente en la zona hilar.

11. Semillas romboidales en sección transversal con dorso suavemente carenado. Pelos largos y abundantes hacia el reborde micropilar, más cortos alrededor de la zona hilar formando un anillo continuo, y escasos sobre la lengüeta hilar

Sida dictyocarpa

11'. Semillas sub-triangulares en sección transversal con dorso plano o suavemente convexo o cóncavo.

12. Reborde micropilar con abundantes pelos Sida rhombifolia

12'. Reborde micropilar glabro.

13. Reborde micropilar notable. Lengüeta hilar glabra Sida variegata

13'. Reborde micropilar pequeño. Lengüeta hilar con pelos escasos. 
Descripciones morfológicas de las diásporas de cada especie

Abutilon pauciflorum A. St.-Hil., Fl. Bras. mer. 1: 161.1827.

Fig. 1a-b

Arbusto perenne nativo. Fruto truncadocilíndrico u ovoide constituido por alrededor de 15 mericarpos dehiscentes, pluriseminados. Mericarpos de aproximadamente 12,5 × 6,0 $\times$ $4,0 \mathrm{~mm}$, agudos en el ápice con dos aristas cortas de alrededor de $1 \mathrm{~mm}$ long. Cara dorsal con carena media prominente por donde se produce la dehiscencia, casi hasta la base cuando maduros, con pubescencia afelpado-estrellada y pelos blancos simples de 1,5-2 mm long., abundantes sobre la zona de dehiscencia. Caras laterales lisas, opacas, papiráceas, sin pelos. Mericarpos sin divisiones interiores. Semillas 2-11, uniseriadas, arriñonadas, de unos $2 \times 1,9 \mathrm{~mm}$, con pelos estrellados dispersos de 2 ramas. Hilo lateral. Zona hilar oscura rodeada por un contorno anular más claro.

Material examinado: Dpto. Santa María, Falda del Carmen, Ea. Las Higuerillas: 31.III.2008, M. Scandaliaris et al. CS 391 (ACOR); M. Scandaliaris et al. CS 427 (ACOR); 11.IV.2008, E. Fuentes et al. CS 602 (ACOR); 25.IV.2008, E. Fuentes et al. CS 646 (ACOR); 28.IV.2008, E. Fuentes et al. CS 692 (ACOR); 24.XI.2008, E. Fuentes et al. CS 951 (ACOR); 11.XII.2008, E. Fuentes et al. CS 1046 (ACOR); 11.III.2010, M.E. Carreras et al. AMP 2733 (ACOR); M.E. Carreras et al. AMP 2743 (ACOR).

Gaya parviflora (Phil.) Krapov, Bonplandia 9 (1-2): 82-83. 1996.

Fig. 1c-e

Hierba o subarbusto perenne nativo. Fruto péndulo, triangular o piramidal, formado por 10-13 mericarpos dehiscentes, uniseminados, membranáceos. Mericarpos de alrededor de 6,2 $\times$ $3,2 \times 1,5 \mathrm{~mm}$, inflados, sin aristas. Cara dorsal de superficie suavemente ondulada, con carena media prominente por donde se produce la dehiscencia hasta la base, con pelos simples dispersos menores de $1 \mathrm{~mm}$ long. Caras laterales transparentes, con nervaduras formando un retículo. Mericarpos con endoglosa ornamentada formada por dos partes: una membranácea, verde, ondulada, y una más dura, dentada, envolviendo y siguiendo la forma de la semilla. Semilla piramidal, de unos $2 \times 1,5$ $\mathrm{mm}$, cubierta de pelos blancos, simples, abundantes en toda la superficie y aún más en la zona del hilo y a lo largo de 5 costillas. Hilo apical. Zona hilar ocupando la tercera parte del largo de la semilla.
Material examinado: Dpto. Santa María, Falda del Carmen, Ea. Las Higuerillas: 7.III.2008, M. Scandaliaris et al. CS 313 (ACOR); M. Scandaliaris et al. CS 321 (ACOR); 17.X.2008, M.E. Carreras et al. CS 811 (ACOR) ; 31.X.2008, J.E. Martinat et al. CS 892 (ACOR); 24.XI.2008, E. Fuentes et al. CS 956 (ACOR); 11.XII.2008, E. Fuentes et al. CS 1034 (ACOR); E. Fuentes et al. CS 1050 (ACOR); 11.III.2010, M.E. Carreras et al. AMP 2745 (ACOR).

Krapovickasia flavescens (Cav.) Fryxell, Brittonia 30 (4): 456. 1978.

Fig. 1f-g

Hierba perenne nativa. Fruto formado por 5 mericarpos indehiscentes, uniseminados, de alrededor de $3,2 \times 2 \times 2 \mathrm{~mm}$, de sección triangular, múticos. Cara dorsal tuberculada, con los márgenes prolongados por una membrana transparente, con escasos pelos simples o glabra. Caras laterales lisas de aspecto ceroso. Semilla péndula, globosa, rugosa, glabra, de unos $2 \times 1,8 \mathrm{~mm}$, muy adherida a la pared del mericarpo. Hilo apical.

Material examinado: ADpto. Santa María, Falda del Carmen, Ea. Las Higuerillas: 7.III.2008, M. Scandaliaris et al. CS 319 (ACOR); 11.IV.2008, E. Fuentes et al. CS 561 (ACOR); 16.V.2008, E. Fuentes et al. CS 750 (ACOR); 17.X.2008, E. Fuentes et al. CS 775 (ACOR); 31.X.2008, M.E. Carreras et al. CS 854 (ACOR); J.E. Martinat et al. CS 893 (ACOR); 14.XI.2008, J.E. Martinat et al. CS 918 (ACOR); 11.III.2010, M.E. Carreras et al. AMP 2746 (ACOR).

Malvastrum coromandelianum (L.) Garcke, Bonplandia 5: 295, 297. $1857 . \quad$ Fig. 1h-i Subarbusto perenne nativo. Fruto formado por alrededor de 12 mericarpos dehiscentes sólo por el margen ventral, uniseminados. Mericarpos de unos $3,2 \times 3 \times 1,8 \mathrm{~mm}$, de sección triangular, provistos de dos aristas laterales horizontales de $0,4 \mathrm{~mm}$ long. y de una arista apical erecta de 1,2 $\mathrm{mm}$. Cara dorsal plana al transcorte; con pelos estrellados abundantes que le otorgan un aspecto afelpado, y pelos simples largos, más abundantes hacia las caras laterales y en las dos aristas horizontales. Caras laterales menos pubescentes, con costillas transversales convergentes hacia el seno ventral y zonas intercostales membranáceas, transparentes. Semilla comprimida, arriñonada, glabra, de alrededor de 1,9 mm long. $\times 1,8 \mathrm{~mm}$ lat. y 1,1 mm de espesor. Hilo lateral. Zona hilar oscura delimitada por un reborde blanquecino.

Material examinado: Dpto. Santa María, Falda del Carmen, Ea. Las Higuerillas: 31.III.2008, M. Scandaliaris et al. CS 454 (ACOR); 12.I.2010, E. Fuentes et al. AMP 2704 (ACOR). 

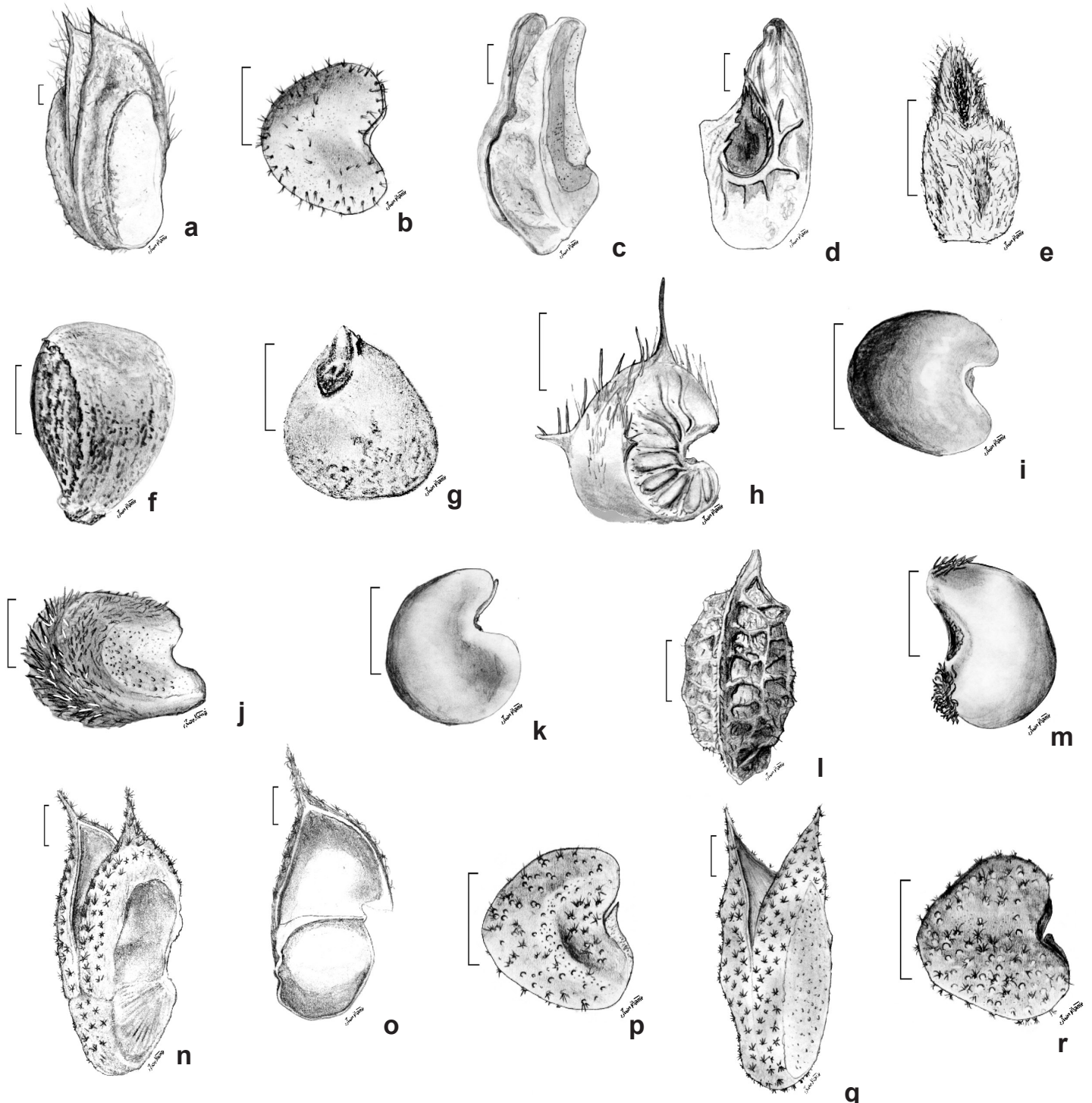

Figura 1 - a-b. Abutilon pauciflorum - a. Mericarpo vista externa. b. Semilla vista lateral. c-e. Gaya parviflora - c. Mericarpo vista externa. d. Mericarpo vista interna. e. Semilla vista hilar. f-g. Krapovickasia flavescens - f. Mericarpo vista externa. g. Semilla vista hilar. h-i. Malvastrum coromandelianum - h. Mericarpo vista externa. i. Semilla vista lateral. j-k. M. interruptum - Mericarpo vista externa. k. Semilla vista lateral. 1-m. Pavonia aurigloba - Mericarpo vista externa. m. Semilla vista lateral. n-p. Pseudabutilon pedunculatum - n. Mericarpo vista externa, o. Mericarpo vista interna. p. Semilla vista lateral. q-r. P. virgatum - q. Mericarpo vista externa. r. Semilla vista lateral. (Escala: $1 \mathrm{~mm}$. Ilustraciones: Juan Viana).

Figure 1 - a-b. Abutilon pauciflorum - a. Mericarp external view. b. Seed lateral view. c-e. Gaya parviflora - c. Mericarp external view. d. Mericarp inner view. e. Seed hilum view. f-g. Krapovickasia flavescens - f. Mericarp external view. g. Seed hilum view. h-i. Malvastrum coromandelianum - h. Mericarp external view. i. Seed lateral view. j-k. M. interruptum - Mericarp external view. k. Seed lateral view. 1-m. Pavonia aurigloba - Mericarp external view. m. Seed lateral view. n-p. Pseudabutilon pedunculatum - n. Mericarp external view, o. Mericarp inner view. p. Seed, lateral view. q-r. P. virgatum - q. Mericarp, external view. r. Seed, lateral view. (Scale: $1 \mathrm{~mm}$. Ilustrations: Juan Viana). 
Malvastrum interruptum K. Schum., Fl. Bras. 12 (3): 272. 1891.

Fig. $1 \mathrm{j}-\mathrm{k}$

Subarbusto perenne nativo. Fruto formado por 10-12 mericarpos dehiscentes sólo por el margen ventral, uniseminados. Mericarpos de unos 2,2 $\times 2$ $\times 0,8 \mathrm{~mm}$, de sección triangular, sin aristas. Cara dorsal convexa, plana o ligeramente cóncava al transcorte, con pelos estrellados cortos, blancos, y pelos simples más largos, o con dos ramas, hacia la cara lateral. Caras laterales lisas, membranáceas. Semilla comprimida, arriñonada, lisa, glabra, de alrededor de 1,5 mm long. $\times 1,2 \mathrm{~mm}$ lat. y $0,8 \mathrm{~mm}$ de espesor. Hilo lateral. Zona hilar clara delimitada por un reborde blanquecino.

Material examinado: Dpto. Santa María, Falda del Carmen, Ea. Las Higuerillas: 11.IV.2008, E. Fuentes et al. CS 503 (ACOR); R. Lovey et al. CS 506 (ACOR); 11.IV.2008, E. Fuentes et al. CS 508 (ACOR); 25.IV.2008, E. Fuentes et al. CS 642 (ACOR); 11.III.2010, M. E. Carreras et al. AMP 2738 (ACOR).

Pavonia aurigloba Krapov. \& Cristóbal, Lilloa 31: 29. 1962.

Fig. 11-m

Arbusto perenne endémico. Fruto formado por 5 mericarpos indehiscentes, uniseminados, óseos. Mericarpos de alrededor de 4,5 × 2,5 $\times$ $2,5 \mathrm{~mm}$, con la superficie externa uniformemente reticulada con celdas profundas y provista de pelos simples, blancos, rígidos, notables. Cara dorsal convexa, con carena media prominente prolongada en un pequeño apículo y caras laterales planas. Semilla arriñonada, lisa, de unos $2 \times 1,5 \mathrm{~mm}$, con 2 mechones de pelos simples, blancos, ondulados, dispuestos por encima y por debajo de la zona hilar oscura y de 2/3 del largo de la semilla. Hilo lateral. Material examinado: Dpto. Santa María, Falda del Carmen, Ea. Las Higuerillas: 31.III.2008, M. Scandaliaris et al. CS 434 (ACOR); 11.IV.2008, E. Fuentes et al. CS 589 (ACOR); 28.IV.2008, E. Fuentes et al. CS 667 (ACOR); 24.XI.2008, E. Fuentes et al. CS 950 (ACOR); 11.XII.2008, E. Fuentes et al. CS 1052 (ACOR); 28.II.2009, E. Fuentes et al. CS 1103 (ACOR); 30.III.2009, M.E. Carreras et al. CS 1126 (ACOR); 11.III.2010, M.E. Carreras et al. AMP 2742 (ACOR).

Pseudabutilon pedunculatum (R. E. Fr.) Krapov., Flora de San Juan 2: 113. $2003 . \quad$ Fig. 1n-p

Subarbusto perenne nativo. Fruto cilíndrico formado por 6-10 mericarpos dehiscentes en el ápice, 3 -seminados. Mericarpos de unos $7 \times 4 \times 2 \mathrm{~mm}$, con 2 aristas de aproximadamente 1/6 del largo total del mericarpo. Cara dorsal estrellado-pubescente, con nervadura media marcada y una línea transversal notable coincidente con la endoglosa interna.
Caras laterales papiráceas, opacas, glabras, con un retículo suave de celdas alargadas en el tercio inferior. Mericarpos divididos en 2 compartimentos superpuestos, separados por una lengüeta dorsal o endoglosa transversal completa; la cavidad inferior uniseminada y la superior biseminada. Semillas de alrededor de 1,8 $\times 1,4 \mathrm{~mm}$, subtriangulares en vista lateral con una zona más clara en forma de arco, con pelos estrellados de más de 3 ramas asociados en grupos otorgando a la superficie un aspecto punteado. Hilo lateral.

Material examinado: Dpto. Santa María, Falda del Carmen, Ea. Las Higuerillas: 31.III.2008, M. Scandaliaris et al. CS 404 (ACOR); M. Scandaliaris et al. CS 409 (ACOR); 31.X.2008, M.E. Carreras et al. CS 857 (ACOR); M.E. Carreras et al. CS 858 (ACOR); M.E. Carreras et al. CS 891 (ACOR); 24.XI.2008, E. Fuentes et al. CS 953 (ACOR).

Pseudabutilon virgatum (Cav.) Fryxell, Contr. Univ. Michigan Herb. 21: 191. 1997.

Fig. 1q-r

Subarbusto perenne nativo. Fruto formado por 8 mericarpos dehiscentes en el ápice, 3-seminados. Mericarpos de unos $7 \times 3,5 \times 2,3 \mathrm{~mm}$, con 2 aristas de aproximadamente 1/6 del largo total del mericarpo. Cara dorsal cubierta de pelos estrellados diminutos, con nervadura media marcada, sin línea transversal. Caras laterales papiráceas, glabras, lisas. Mericarpos sin endoglosa, con una sola cavidad 3-seminada. Semillas de alrededor de 1,8 $\times 1,8 \mathrm{~mm}$, subtriangulares en vista lateral, de color uniforme, con pelos estrellados de más de 3 ramas asociados en grupos otorgando a la superficie un aspecto punteado. Hilo lateral.

Material examinado: Dpto. Santa María, Falda del Carmen, Ea. Las Higuerillas: 31.III.2008, M. Scandaliaris et al. CS 404 (ACOR); M. Scandaliaris et al. CS 409 (ACOR); 31.X.2008, M.E. Carreras et al. CS 857 (ACOR); M.E. Carreras et al. CS 858 (ACOR); M.E. Carreras et al. CS 891 (ACOR); 24.XI.2008, E. Fuentes et al. CS 953 (ACOR).

Sida argentina K. Schum., Fl. Bras. 12 (3): 315. 1891. Fig. 2a-b

Hierba perenne nativa. Fruto formado por 5 mericarpos dehiscentes en el ápice, uniseminados. Mericarpos de unos $4 \times 2 \times 2 \mathrm{~mm}$, trígonos, con dos aristas verticales cortas de alrededor de $0,5-1$ $\mathrm{mm}$ de largo. Cara dorsal y laterales con la mitad inferior con un retículo de celdas profundas, membranáceas, más o menos redondeadas y rebordes notables, ondulados, cubiertas con pelos simples dispersos más largos en la zona de dehiscencia otorgándole un aspecto ciliado. Cara 

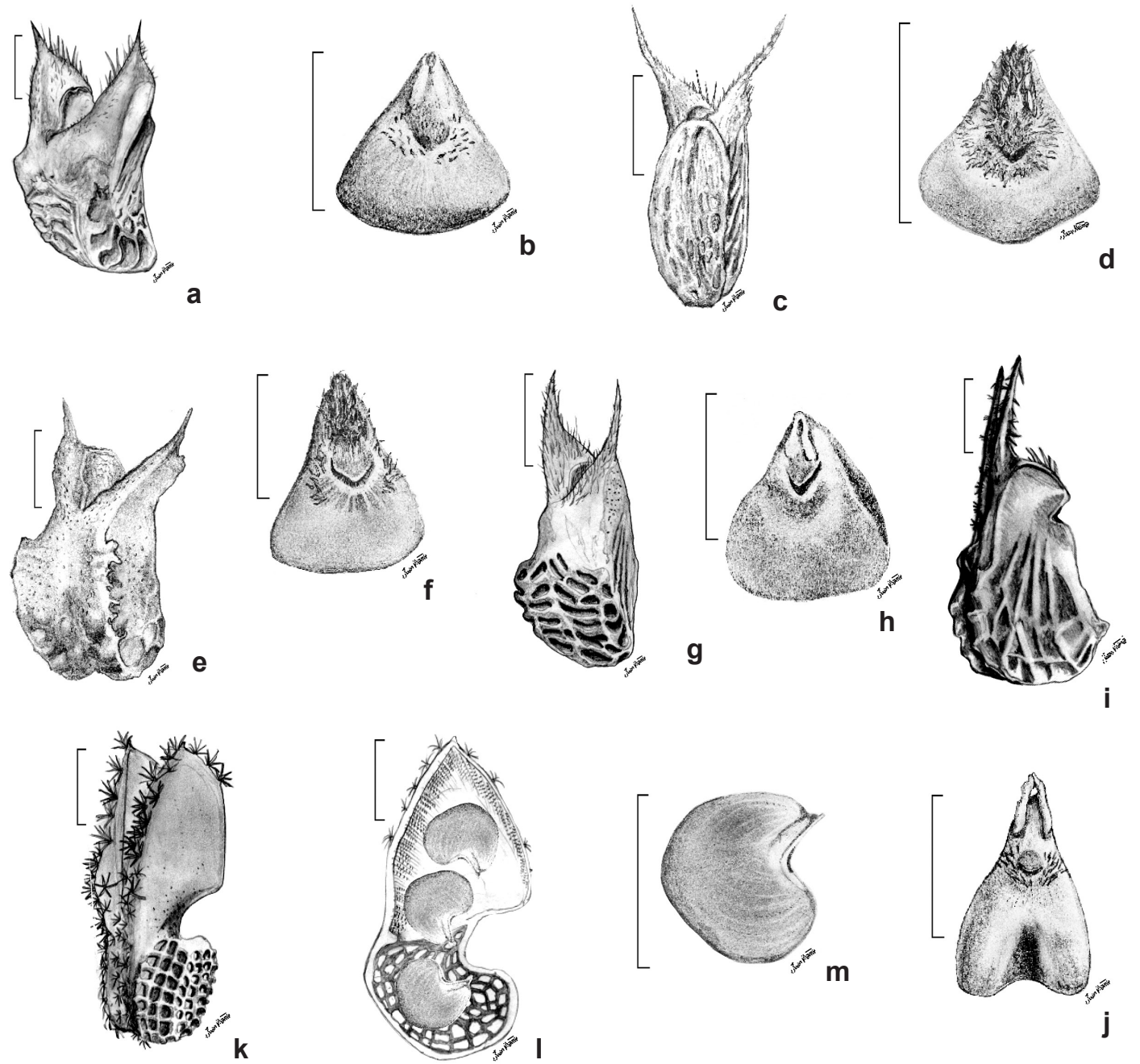

Figura 2 -a-b. Sida argentina - a. Mericarpo vista externa. b. Semilla vista hilar. c-d. S. dictyocarpa-Mericarpo vista externa. d. Semilla vista hilar. e-f. S. rhombifolia - Mericarpo vista externa. f. Semilla vista hilar. g-h. S. spinosa - Mericarpo vista externa. h. Semilla vista hilar. i-j. S. variegata -i. Mericarpo vista externa. j. Semilla vista hilar. k-m. Sphaeralcea cordobensis - k. Mericarpo vista externa. 1. Mericarpo vista interna. m. Semilla vista lateral. (Escala: $1 \mathrm{~mm}$. Ilustraciones: Juan Viana). Figure 2 - a-b. Sida argentina - a. Mericarp external view. b. Seed hilum view. c-d. S. dictyocarpa - Mericarp external view. d. Seed hilum view. e-f. S. rhombifolia - Mericarp external view. f. Seed hilum view. g-h. S. spinosa-Mericarp external view. h. Seed hilum view. i-j. S. variegata-i. Mericarp external view. j. Seed hilum view. k-m. Sphaeralcea cordobensis - k. Mericarp external view. 1. Mericarp inner view. m. Seed lateral view. (Scale: 1 mm. Ilustrations: Juan Viana).

dorsal subtriangular oblicua, con el extremo basal terminado en punta. Semilla triangular en sección transversal con dorso convexo, de alrededor de 1,8 $\times 1 \mathrm{~mm}$, de superficie glabra. Hilo apical. Zona hilar rodeada de pelos, lengüeta hilar oscura con pelos escasos y reborde micropilar claro en forma de v, glabro.

Material examinado: Dpto. Santa María, Falda del Carmen, Ea. Las Higuerillas: 31.III.2008, M. Scandaliaris et al. CS 413 (ACOR); M. Scandaliaris et al. CS 471 (ACOR); 17.X.2008, E. Fuentes et al. CS 768 (ACOR);
14.XI.2008, J.E. Martinat et al. CS 903 (ACOR); J.E. Martinat et al. CS 917 (ACOR); J.E. Martinat et al. C.S. 919 (ACOR); 11.XII.2008, E. Fuentes et al. CS 1047 (ACOR); E. Fuentes et al. CS 1051 (ACOR).

Sida dictyocarpa Griseb. ex K. Schum., Fl. Bras. 12 (3): 314. 1891.

Fig. 2c-d

Subarbusto perenne nativo. Fruto formado por 5 mericarpos dehiscentes en el ápice, uniseminados. Mericarpos de unos $3 \times 1 \times 1,1 \mathrm{~mm}$, trígonos, con dos aristas verticales de $1-1,5 \mathrm{~mm}$ de largo. Cara dorsal 
y laterales con los $2 / 3$ basales reticuladas, con pelos simples dispersos, más largos en la zona de dehiscencia. Cara dorsal ovalado convexa, recorrida por un nervio medio longitudinal marcado y celdas del retículo redondeadas. Caras laterales papiráceas, tenues, retículos con celdas alargadas. Semilla romboidal en sección transversal con dorso suavemente carenado, de alrededor de 1,8 $\times 1 \mathrm{~mm}$. Hilo apical. Pelos largos y abundantes hacia el reborde micropilar, más cortos alrededor de la zona hilar formando un anillo continuo, y escasos sobre la lengüeta hilar oscura.

Material examinado: Dpto. Santa María, Falda del Carmen, Ea. Las Higuerillas: 7.III.2008, M. Scandaliaris et al. CS 334 (ACOR); 31.III.2008, M. Scandaliaris et al. CS 393 (ACOR); 11.IV.2008, E. Fuentes et al. CS 487 (ACOR); 25.IV.2008, E. Fuentes et al. CS 635 (ACOR); 16.V.2008, E. Fuentes et al. CS 748 (ACOR); 24.XI.2008, E. Fuentes et al. CS 955 (ACOR); 11.XII.2008, E. Fuentes et al. CS 1021 (ACOR); 28.II.2009, E. Fuentes et al. CS 1101 (ACOR); 11.III.2010, M.E. Carreras et al. AMP 2748 (ACOR).

Sida rhombifolia L., Sp. Pl. 2: 684. 1753.

Fig. 2e-f

Hierba o subarbusto, anual o perenne, nativo. Fruto formado por 10-14 mericarpos dehiscentes en el ápice, uniseminados. Mericarpos de alrededor de $3,5 \times 2,5 \times 1,4 \mathrm{~mm}$, trígonos, comprimidos, con dos aristas de aproximadamente $2 \mathrm{~mm}$ de largo dirigidas hacia ventral. Cara dorsal y laterales con la mitad inferior reticulada, con pelos simples y estrellados escasos, en la zona de dehiscencia de igual largo que los de las aristas. Cara dorsal rectangular, con un suave retículo de celdas sobre-elevadas. Caras laterales tan anchas como largas, con un tenue reticulado de celdas redondeadas más notables en el margen. Semilla triangular en sección transversal con dorso plano-convexo, de unos $2 \times 1,6 \mathrm{~mm}$. Hilo apical. Pelos a los costados de la zona hilar; lengüeta hilar oscura, glabra; reborde micropilar claro, en forma de $\mathrm{v}$, desarrollado, con pelos abundantes. Material examinado: Dpto. Santa María, Falda del Carmen, Ea. Las Higuerillas: 31.III.2008, M. Scandaliaris et al. CS 407 (ACOR); 11.IV.2008, E. Fuentes et al. CS 509 (ACOR); 25.IV.2008, E. Fuentes et al. CS 626 (ACOR); 28.IV.2008, E. Fuentes et al. CS 669 (ACOR); 16.V.2008, E. Fuentes et al. CS 749 (ACOR); 17.X.2008, E. Fuentes et al. CS 812 (ACOR); 24.XI.2008, E. Fuentes et al. CS 961 (ACOR); 11.XII.2008, E. Fuentes et al. CS 1048 (ACOR); 11.III.2010, M.E. Carreras et al. AMP 2744 (ACOR).

Sida spinosa L., Sp. Pl. 2: 683. 1753.

Fig. 2g-h

Subarbusto perenne nativo. Fruto formado por 5 mericarpos, dehiscentes en el ápice, uniseminados.
Mericarpos de unos 3,5 × 1,8 × 1,9 mm, trígonos, con dos aristas de alrededor de 1,5 $\mathrm{mm}$ de largo. Caras dorsal y laterales reticuladas, con pelos simples notables en la zona de dehiscencia y en las aristas. Cara dorsal subtriangular oblicua con el extremo basal terminado en punta, reticulada en la base con aspecto estriado transverso. Caras laterales con retículo de celdas redondeadas hacia dorsal y alargadas hacia ventral. Semilla triangular en sección transversal con dorso plano-convexo, de alrededor de 1,8 $\times 1,1 \mathrm{~mm}$, glabra. Hilo apical. Zona hilar-micropilar sin pelos. Material examinado: Dpto. Santa María, Falda del Carmen, Ea. Las Higuerillas: 31.III.2008, M. Scandaliaris et al. CS 392 (ACOR); M. Scandaliaris et al. CS 440 (ACOR); 11.IV.2008, E. Fuentes et al. CS 518 (ACOR); E. Fuentes et al. CS 527 (ACOR); 28.II.2009, E. Fuentes et al. CS 1102 (ACOR); 11.III.2010, M. E. Carreras et al. AMP 2747 (ACOR); M.E. Carreras et al. AMP 2739 (ACOR); M.E. Carreras et al. AMP 2747 (ACOR).

Sida variegata (Griseb.) Krapov., Bol. Soc. Argent. Bot. 4 (3): 187. 1952.

Fig. 2i-j

Hierba o subarbusto perenne nativo. Fruto formado por 12 mericarpos dehiscentes en el ápice, uniseminados. Mericarpos de alrededor de 4,5 $\times 2 \times$ $1,2 \mathrm{~mm}$, trígonos, con dos aristas apicales verticales. Cara dorsal y laterales reticuladas con celdas transparentes y rebordes claros, con pelos estrellados abundantes en la parte apical del dorso y en aristas, y pelos simples asociados con la zona de dehiscencia y retrorsos en las aristas. Cara dorsal con retículo de celdas profundas y rebordes ondulados. Caras laterales con retículo de celdas transparentes, alargadas hacia ventral, con un pico en la base del margen ventral. Semilla triangular en sección transversal con dorso cóncavo en su parte media, de unos 2,2 × 1,6 mm. Hilo apical. Zona hilar con pelos más abundantes por debajo del hilo, lengüeta hilar oscura glabra, reborde micropilar en forma de v, claro, glabro, prolongándose más allá del extremo de la semilla.

Material examinado: Dpto. Santa María, Falda del Carmen, Ea. Las Higuerillas: 11.III.2010, M.E. Carreras et al. AMP 2741 (ACOR); M.E. Carreras et al. AMP 2736 (ACOR).

Sphaeralcea cordobensis Krapov., Lilloa 17: 214216. 1949.

Fig. 2k-m

Subarbusto perenne endémico. Fruto formado por 14 a 16 mericarpos con dehiscencia apical, 2-3-seminados. Mericarpos de unos 4,1 $\times 2 \times 0,8 \mathrm{~mm}$, comprimidos, con dos aristas en forma de breves mucrones apicales de $0,1 \mathrm{~mm}$, 
a veces ausentes. Cara dorsal angosta, cubierta totalmente por pelos estrellados abundantes, más largos en el ápice. Caras laterales membranáceas, transparentes, con un retículo de celdas redondeadas en el tercio inferior. Mericarpos divididos en 2 compartimentos por una pequeña lengüeta dorsal o endoglosa incompleta. Semillas lenticulares, de alrededor de $1 \times 0,8 \mathrm{~mm}$, con escasos pelos simples. Hilo lateral.

Material examinado: Dpto. Santa María, Falda del Carmen, Ea. Las Higuerillas: 11.III.2010, M.E. Carreras et al. AMP 2734 (ACOR).

Este trabajo contribuye al conocimiento, análisis e identificación de especies de Malvaceae, presentes en el banco de semillas del suelo de zonas serranas afectadas por incendios en la provincia de Córdoba, Argentina, y constituye un aporte para los estudios de regeneración natural y mantenimiento de la riqueza florística, tendientes a comprender o predecir la recuperación de la vegetación nativa y las posibilidades de su conservación.

\section{Agradecimientos}

Agradecemos al Ing. Agr. Juan Viana por las ilustraciones y a la Facultad de Ciencias Agropecuarias de la UNC por haber facilitado el vehículo con el que se hicieron los viajes de relevamiento y recolección. Este trabajo fue financiado por la Secretaría de Ciencia y Tecnología de la Universidad Nacional de Córdoba (SECYT, UNC).

\section{Referencias}

Abril, A. \& González, C. 1999. Dinámica de la fertilidad y de las poblaciones microbianas en suelos afectados por incendios en las sierras de Córdoba (Argentina). Agriscientia 16: 63-70.

Arias Toledo, B. 2009. Diversidad de usos, prácticas de recolección y diferencias según género y edad en el uso de plantas medicinales en Córdoba, Argentina. Boletín Latinoamericano y del Caribe de Plantas Medicinales y Aromáticas 8: 389-401.

Atala, D.; Baudo, F.; Garré, S.; Miatello, R.; Juárez, G. \& Fernández, F. 2005. Informe de inspección y evaluación económica del incendio de Villa Carlos Paz. Agencia Córdoba Ambiente S.E., Córdoba. 31p.

Barrionuevo, V. 2008. Evaluación del potencial ornamental de especies nativas herbáceas y subarbustivas del valle de Paravachasca (Córdoba, Argentina). Tesis de Maestría. Universidad Nacional de Córdoba, Córdoba. 244p.
Bayer, C.; Fay, M.F.; Bruijen, A.Y.D.; Savolainen, V.; Morton, C.M.; Kubitzki, K.; Alberson, W.S. \& Chase, M.W. 1999. Support for an exanded family concept of Malvaceae within a recircumscribed order Malvales: a combined analysis of plastid atpB and $r b c L$ DNA sequences. Botanical Journal of the Linnean Society 129: 267-303.

Bayer, C. \& Kubitzki, K. 2003. Malvaceae. In: Kubitzki, K. \& Bayer, C. (ed.). Flowering plants, dicotyledons: Malvales, Capparales and nonbetalain Caryophyllales. Springer-Verlag, Berlin. Pp. 225-311.

Bianco, C.; Nuñez, C. \& Graus, T. 2000. Identificación de frutos y semillas de las principales malezas del centro de Argentina. Editorial de la Fundación Universidad Nacional de Río Cuarto, Córdoba. 99p.

Carreras, M.E.; Ruiz, G.M.; \& Bossa, S.R. 2008. Plantas nativas con posible valor ornamental. Aplicación Multimedia interactiva. CD ROM. SIMA Ed., Córdoba.

Carreras, M.E.; Fuentes, E.; Martinat, J.E.; Bossa, S.R. \& Carbone, L. 2009. Aportes para el análisis del banco de semillas del suelo de zonas serranas (Córdoba) afectadas por Incendios II. Clave dicotómica para identificar especies de Malvaceae. Boletín de la Sociedad Argentina de Botánica 44(supl.): 82-83.

Castillo Carrillo, P.S.; Carranza, S. \& Villalba, K. 2006. Entomofauna asociada a Malvastrum coromandelianum (L.) Garcke (Malvales: Malvaceae), una maleza productora de néctar. En Memoria I Reunión de Ciencia y Tecnología Agraria en el Norte de Perú. Innovación y Competitividad para el Agro Peruano (INCAGRO). Ministerio de Agricultura (MINAG). 22p.

Díaz-Delgado, R. 2003. Efecto de la recurrencia de los incendios sobre la resiliencia post-incendio de las comunidades vegetales de Cataluña a partir de imágenes de satélite. Ecosistemas XII(3): 0 Disponible en $<$ http://redalyc.uaemex.mx/redalic/ sra/inicio/ArtPdfRED.jsp? ICue $=54012304 \mathrm{IS}$ SN1132-6344>. Acceso en 15 Abr 2011.

Estadística de Incendios Forestales. 2008. Publicación de la Secretaría de Ambiente y Desarrollo Sustentable de la Nación, Ciudad Autónoma de Buenos Aires. 107p.

Estadística de Incendios Forestales. 2009. Publicación de la Secretaría de Ambiente y Desarrollo Sustentable de la Nación, Ciudad Autónoma de Buenos Aires. $104 \mathrm{p}$.

Ferri, R.; Ceballos, M.; Vischi, N.; Heredia, E. \& Oggero, A. 2009. Banco de semillas de un relicto de Espinal (Córdoba, Argentina). Iheringia 64: 93-100.

Fryxell, P.A. 1999. Pavonia Cavanilles (Malvaceae). Flora Neotropica Monographs 76: 2-284.

Fuentes, E.; Carreras, M.E.; Loyola, M.J.; Martinat, J.E. \&. Jewsbury, G. 2009a. Aportes al análisis 
del banco de semillas del suelo de zonas serranas (Córdoba) afectadas por Incendios III. Clave para la identificación de especies de Asteraceae. Boletín de la Sociedad Argentina de Botánica 44(supl.): 88-89.

Fuentes, E.; Carreras, M.E.; Martinat, J.E.; Jewsbury, G. \& Lovey, R.J. 2009b. Composición florística postincendio en zonas serranas de Falda del Carmen (Córdoba, Argentina). I Jornadas Patagónicas de Biología. III Jornadas Estudiantiles de Ciencias Biológicas. Libro de resúmenes: 58. Universidad Nacional de la Patagonia San Juan Bosco. Trelew, Chubut.

Fuentes, E.; Carreras, M.E.; Martinat, J.E.; Jewsbury, G.; Loyola, M.J. \& Carbone, L. 2010. Especies de valor forrajero en flora serrana posfuego de Córdoba-Argentina. X Congreso Latinoamericano de Botánica. "Conservación y uso sustentable de la flora nativa latinoamericana” CD pdf Resúmenes 78. La Serena.

Hinsley, S.R. 2006. Classification of Malvaceae: Overview, compostion: Position: Division. Malvaceae Info (Home). Net Page.

Krapovickas, A. 1949. Las especies de Sphaeralcea de Argentina y Uruguay. Lilloa 17: 179-222.

Krapovickas, A. 1957. Las especies de Malvastrum Sect. Malvastrum de la Flora Argentina. Lilloa 28: 181-195.

Krapovickas, A. 1996. Sinopsis del género Gaya (Malvaceae). Bonplandia 9: 57-87.

Krapovickas, A. 1999. Malvaceae. Monographs in Systematic Botany from the Missouri Botanical Garden 74: 813-844.

Krapovickas, A. 2005. Malvaceae. In: Burkart, A. \& N. M. Bacigalupo. Flora ilustrada de Entre Ríos. Vol. 6. Colección Científica del INTA, Buenos Aires. Pp. 265-333.

Krapovickas, A. 2006. Las especies argentinas y de países vecinos de Sida secc. Nelavaga (Malvaceae, Malveae). 1 Bonplandia 15: 5-45.

Krapovickas, A. 2008. Malvaceae. Monographs in Systematic Botany from the Missouri Botanical Garden 107: 2463-2520.

Krapovickas, A. \& Cristóbal, C.L. 1962. Notas sobre la sección Lebretonia. Pavonia (Malvaceae) y revisión de las especies argentinas. Lilloa 31: 5-73.

López Martínez, N. 2009. Malezas asociadas a plantas ornamentales. Fitosanidad 13: 233-235.

Luti, R.; Bertrán de Solís, M.A.; Galera, F.M.; Müller de Ferreira, N.; Berzal, M.; Nores, M.; Herrera, M.A. \&. Barrera, J.C. 1979. Vegetación. In: Vázquez, J.B.; Miatello, R.A. \& Roqué, M.E. (directores). Geografía física de la Provincia de Córdoba. Editorial Boldt. Buenos Aires. Pp. 297-368.
Martin, A. C. \& Barkley, W. D. 1973. Seed identification manual. University of California Press, Berkeley, Los Angeles and London. 221p.

Martinat, J.E.; Lovey, R.J.; Carreras, M.E. \& Fuentes, E. 2009. Análisis del banco de semillas del suelo en zonas serranas (Falda del Carmen, Córdoba) afectadas por incendios I. Clave para la identificación de especies de Fabáceas. I Jornadas Patagónicas de Biología y III Jornadas Estudiantiles de Ciencias Biológicas. Libro de resúmenes. Universidad Nacional de la Patagonia San Juan Bosco. Trelew, Chubut. 83p.

Martínez Carretero, E. 1995. Los incendios forestales en la Argentina. Multequina 4: 105-114.

Martínez, G. 2005. Recolección y Comercialización de Plantas Medicinales en el Departamento Santa María, Provincia de Córdoba, Argentina. Acta Farmacéutica Bonaerense 24: 575-584.

Medrano, C.; Figueroa, V.; Gutiérrez, W.; Villalobos, Y.; Amaya, L. \& Semprúm, E. 1999. Estudio de las malezas asociadas a plantaciones frutales en la planicie de Maracaibo. Venezuela. Revista de la Facultad de Agronomía (LUZ) 16: 583-596.

Meyer, D.J. \& Effenberger, J. 2007. California noxious weed pest. Propagules Identification Manual. California Department of Food \& Agriculture. Plant Pest Diagnostics Center, Sacramento. 88p.

Montenegro, R. 2010. Peligran los bosques en Córdoba, Argentina. RENACE Red Nacional de Acción Ecologista. Argentina. Disponible en $<$ http://www. renace.net/spip.php?article330>. Acceso en 09 Mayo 2012.

Petetin, C. \& Molinari, E. 1982. Reconocimiento de semillas de malezas. Colección Científica del INTA, Buenos Aires. 146p.

Piudo, M.J. \& Cavero, R.Y. 2005. Banco de semillas: comparación de metodologías de extracción, de densidad y de profundidad de muestreo. Publicaciones de Biología, Universidad de Navarra, Serie Botánica 16: 71-85.

Torres Y.A.; Long, M.A. \& Zalba, S.M. 2008. Reproducción de Pavonia cymbalaria (Malvaceae), una especie nativa con potencial ornamental. Phyton 77: 151-160.

Verzino, G; Joseau, J.; Dorado, M.; Gellert, E.; Rodríguez Reartes, S. \& Nóbile, R. 2005. Impacto de los incendios sobre la diversidad vegetal, sierras de Córdoba, Argentina. Ecología Aplicada 4: 25-34.

Zuloaga, F.O.; Morrone, O. \& Belgrano, M.J. (eds.). 2008. Catálogo de plantas vasculares del Cono Sur (Argentina, Sur de Brasil, Chile, Paraguay y Uruguay). Monographs in Systematic Botany from the Missouri Botanical Garden 107: 3-348. Ed. Missouri Botanical Garden Press. 